\title{
On the Connected Safe Number of Some Classes of Graphs
}

\author{
Rakib Iqbal, ${ }^{1}$ Muhammad Shoaib Sardar $\mathbb{D}^{2},{ }^{2}$ Dalal Alrowaili $\left(\mathbb{D},{ }^{3}\right.$ Sohail Zafar, \\ and Imran Siddique ${ }^{1}{ }^{1}$
}

${ }^{1}$ Department of Mathematics, University of Management and Technology, Lahore 54770, Pakistan

${ }^{2}$ School of Mathematics, Minhaj University, Lahore, Pakistan

${ }^{3}$ Mathematics Department, College of Science, Jouf University, P.O. Box: 2014, Sakaka, Saudi Arabia

Correspondence should be addressed to Imran Siddique; imransmsrazi@gmail.com

Received 9 September 2021; Accepted 7 October 2021; Published 23 October 2021

Academic Editor: Muhammad Kamran Siddiqui

Copyright (c) 2021 Rakib Iqbal et al. This is an open access article distributed under the Creative Commons Attribution License, which permits unrestricted use, distribution, and reproduction in any medium, provided the original work is properly cited.

For a connected simple graph $\mathscr{G}$, a nonempty subset $\mathcal{S}$ of $V(\mathscr{G})$ is a connected safe set if the induced subgraph $\mathscr{G}[\mathcal{S}]$ is connected and the inequality $|\mathcal{S}| \geq|\mathscr{D}|$ satisfies for each connected component $\mathscr{D}$ of $\mathscr{G} \backslash \mathcal{S}$ whenever an edge of $\mathscr{G}$ exists between $\mathcal{S}$ and $\mathscr{D}$. A connected safe set of a connected graph $\mathscr{G}$ with minimum cardinality is called the minimum connected safe set and that minimum cardinality is called the connected safe numbers. We study connected safe sets with minimal cardinality of the ladder, sunlet, and wheel graphs.

\section{Introduction}

A facility location problem (FLP) means to place and manage a certain facility in such a way as to get or achieve the maximum objective with minimizing cost. For further study of FLPs, we refer to the literature of combinatorial optimization [1]. Fujita et al. [2] studied the FLP and proposed a notion of a safe set of graphs.

We refer to [3] for terminology and notation not explained here. Throughout the paper, we will consider only simple and connected graphs. Let $\mathscr{G}$ be a graph with $V(\mathscr{G})$ and $E(\mathscr{G})$ as its vertex and edge set, respectively. The number of vertices in a graph $\mathscr{G}$ is the order of $\mathscr{G}$. For $v \in V(\mathscr{G}), \quad N(v)=\{u \in V(\mathscr{G}): u$ is adjacent to $v\} \quad$ and $N[v]=N(v) \cup\{v\}$ is called open and closed neighborhood of $v$ in $\mathscr{G}$, respectively. For $v \in V(\mathscr{G})$, the degree of vertex $v$ is defined as $\operatorname{deg}(v)=|N(v)|$. For subset $\mathcal{S} \subset V(\mathscr{G}), \mathscr{G}[\mathcal{S}]$ denotes the subgraph induced by $\mathcal{S}$. For subset $X \subset V(\mathscr{G})$, if $\mathscr{G} \backslash X$ is disconnected, then $X$ is known as vertex cut. The vertex connectivity denoted by $\kappa(\mathscr{G})$ is defined as $\min \{|X|: X$ is vertex cut $\}$. Let $C(\mathscr{G})$ denote the set of all connected components of $\mathscr{G}$.

Suppose $A$ and $B$ are disjoint subgraphs of $\mathscr{G}$; then, the set of edges of $E(\mathscr{G})$ that joins some vertices of $A$ and $B$ is denoted by $E(A, B)$. A nonempty subset $\mathcal{S}$ of $V(\mathscr{G})$ is a safe set if, for each $X \in C(\mathscr{G} \backslash \mathcal{S})$ and each $Y \in C(\mathscr{G}[\mathcal{S}])$, the inequality $|Y| \geq|X|$ holds whenever $E(X, Y) \neq \varnothing$. If $\mathscr{G}[\mathcal{S}]$ is connected, then $\mathcal{S}$ is known as a connected safe set. For any graph $\mathscr{G}, s(\mathscr{G})=\min \{|\delta|: \mathcal{S}$ is a safe set of $\mathscr{G}\}$ and $c s(\mathscr{G})=\min \{|\mathcal{S}|: \mathcal{S}$ is a connected safe set of $\mathscr{G}\}$ are known as the safe number and connected safe number, respectively. It is clear from the definition that $s\left(P_{n}\right)=$ $c s\left(P_{n}\right)=\lceil n / 3\rceil$ and $s\left(C_{n}\right)=c s\left(C_{n}\right)=\lceil n / 2\rceil$, where $P_{n}$ and $C_{n}$ are the path and cycle of order $n$, respectively. Fujita et al. [2] proved that for any graph $\mathscr{G}, s(\mathscr{G}) \leq$ $c s(\mathscr{G}) \leq 2 s(\mathscr{G})-1$.

In general, there is no algorithm available to compute a safe number and a connected safe number of a graph $\mathscr{G}$. It was shown in [2] that the computation of safe number and connected safe number is an NP-complete problem. But, on the contrary, Fujita et al. [2] show that $\operatorname{cs}(\mathscr{G})$ can be computed in linear time in case of trees. Also, Árueda et al. [4] show that $s(\mathscr{G})$ can be computed in $O\left(n^{5}\right)$ time on trees. Any tree $T$ with one vertex of degree not more than 3 holds that $s(T)=c s(T)$. Motivated by this equality, for the Cartesian product of two complete graphs, say $\mathscr{G}$, Kang et al. [5] proved that the safe number $s(\mathscr{G})$ and connected safe number $\operatorname{cs}(\mathscr{G})$ are also the same. 
For a vertex-weighted graph, Bapat et al. [6] presented the weighted safe set problem by considering the graph as a community network. For further study about the weighted safe set, we refer to [7-9]. Furthermore, the study on safe set and weighted safe set was conducted by several authors. The parameterized complexity of safe set problems was investigated by Belmonte et al. [10]. Macambiraa et al. [11] presented a mixed integer linear programming formulation and an algorithm for both the weighted safe set and the safe set problems. Fujita and Furuya [12] investigated the comparison between integrity and the safe number of graphs.
In this paper, we describe the connected safe set and compute the connected safe number of ladder, wheel, and sunlet graphs, respectively. The paper is concluded in Section 5.

\section{Safe Set of the Ladder Graph}

For convenience, we consider the ladder graph $\mathscr{G}$ of order $n \geq 4$ with vertex set $V(\mathscr{G})=\left\{v_{1}, v_{2}, \ldots, v_{n}\right\}$ labeled as shown in Figure 1 [13].

Theorem 1. Let $\mathscr{G}$ be a ladder graph; then, the connected safe set is

$$
\mathcal{S}= \begin{cases}\left\{v_{\lfloor n / 8\rfloor+2}, v_{\lfloor n / 8\rfloor+3}, \ldots, v_{\lfloor n / 8\rfloor\lfloor n / 4\rfloor+1}, v_{n\lfloor n / 8\rfloor\lfloor n / 4\rfloor}, v_{n\lfloor n / 8\rfloor-1}\right\}, & \text { if } n \equiv 0,2,6(\bmod 8) \\ \left\{v_{\lfloor n / 8\rfloor+2}, v_{\lfloor n / 8\rfloor+3}, \ldots, v_{\lfloor n / 8\rfloor\lfloor n / 4\rfloor}, v_{n\lfloor n / 8\rfloor\lfloor n / 4\rfloor+1}, v_{n\lfloor n / 8\rfloor-1}\right\}, & \text { otherwise. }\end{cases}
$$

Proof 1. The proof is divided into two cases:

Case 1: assume that $n \equiv 0,2,6(\bmod 8)$. Let $\mathcal{S}=\left\{v_{\lfloor n / 8\rfloor+2}, v_{\lfloor n / 8\rfloor+3}, \ldots, v_{\lfloor n / 8\rfloor+\lfloor n / 4\rfloor+1}, v_{n-\lfloor n / 8\rfloor-\lfloor n / 4\rfloor}\right.$, $\left.v_{n-\lfloor n / 8\rfloor-1}\right\}$ be a subset of $V(\mathscr{G})$. Clearly, $v_{1} v_{n}$, $v_{2} v_{n-1}, \ldots, v_{n / 2} v_{(n / 2)-1} \in E(\mathscr{G})$. Hence, $v_{\lfloor n / 8\rfloor+2}$ and $v_{n-\lfloor n / 8\rfloor-1}$ as well as $v_{\lfloor n / 8\rfloor+\lfloor n / 4\rfloor+1}$ and $v_{n-\lfloor n / 8\rfloor-\lfloor n / 4\rfloor}$ are adjacent. Therefore, $\mathscr{G}[\mathcal{S}]$ is connected. Now, $C(\mathscr{G} \backslash \mathcal{S})=\left\{\mathscr{D}_{1}, \mathscr{D}_{2}, \mathscr{D}_{3}\right\}$, where $\mathscr{D}_{1}=\left\{v_{n-\lfloor n / 8\rfloor-\lfloor n / 4\rfloor+1}\right.$, $\left.v_{n-\lfloor n / 8\rfloor-\lfloor n / 4\rfloor+2}, \ldots, v_{n-\lfloor n / 8\rfloor-2}\right\}, \quad \mathscr{D}_{2}\left\{v_{\lfloor n / 8\rfloor+\lfloor n / 4\rfloor+2}\right.$, $\left.v_{\lfloor n / 8\rfloor+\lfloor n / 4\rfloor+3}, \ldots, v_{n-\lfloor n / 8\rfloor-\lfloor n / 4\rfloor-1}\right\}$, and $\mathscr{D}_{3}=\left\{v_{1}, v_{2}, \ldots\right.$, $\left.v_{\lfloor n / 8\rfloor+1}, v_{n-\lfloor n / 8\rfloor}, \ldots, v_{n-1}, v_{n}\right\}$. It is easy to see that $|\mathcal{S}|=\lfloor n / 4\rfloor+2, \quad\left|\mathscr{D}_{1}\right|=\lfloor n / 4\rfloor-2, \quad\left|\mathscr{D}_{2}\right|=n-2\lfloor n / 8\rfloor$ $-2\lfloor n / 4\rfloor-2$, and $\left|\mathscr{D}_{3}\right|=2\lfloor n / 8\rfloor+2$. This implies $\left|\mathscr{D}_{i}\right| \leq|\mathcal{S}|$ and $E\left(\mathscr{D}_{i}, \mathcal{S}\right) \neq \varnothing$ for $1 \leq i \leq 3$. Hence, both the conditions of connected safe set are satisfied.

Case 2: the similar arguments might work for the other case.

Theorem 2. For a ladder graph $\mathscr{G}$ of order $n$, the following holds:

$$
\operatorname{cs}(\mathscr{G})= \begin{cases}\left\lceil\frac{n}{3}\right\rceil, & \text { if } 4 \leq n \leq 8, \\ \left\lfloor\frac{n}{4}\right\rfloor+2, & \text { if } n \geq 10 \text { and } n \equiv 0,2,6(\bmod 8), \\ \left\lfloor\frac{n}{4}\right\rfloor+1, & \text { otherwise. }\end{cases}
$$

Proof 2. Assume that $\mathcal{S}$ is a connected safe set of cardinality $\operatorname{cs}(\mathscr{G})$ and $C(\mathscr{G} \backslash \mathcal{S})=\left\{\mathscr{D}_{1}, \mathscr{D}_{2}, \ldots, \mathscr{D}_{t}\right\}$, ordered so that $\left|\mathscr{D}_{1}\right| \leq\left|\mathscr{D}_{2}\right| \leq \cdots \leq\left|\mathscr{D}_{t}\right|$.

If $t=1$, then either $v_{1}, v_{n} \in \mathcal{S}$ or $v_{n / 2}, v_{n / 2+1} \in \mathcal{S}$. We assume that $v_{1}, v_{n} \in \mathcal{S}$. Let $u \in \mathscr{D}_{1}$ such that $E(\{u\}, \mathcal{S}) \neq \varnothing$. Then, by removing vertex $v_{1}$ from $\mathcal{S}$ and adding vertex $u$ in
$\mathcal{S}$, we get a connected safe set $\mathcal{S}^{*}$ and for $\mathcal{S}^{*}$, $\left|\mathscr{D}_{1}\right|<\max \left\{\mid \mathscr{D} \| \mathscr{D} \in C\left(\mathscr{G} \backslash \mathcal{S}^{*}\right)\right\}$, which is a contradiction. Hence, $t \geq 2$.

Consider that $n \geq 10$ and $n \equiv 0,2,6(\bmod 8)$.

We want to show that $|\mathcal{S}|=\lfloor n / 4\rfloor+2$. Suppose on the contrary $|\mathcal{S}|=\lfloor n / 4\rfloor+1$ since $\kappa(\mathscr{G})=2$.

Let $\mathscr{X}=\left\{\left\{v_{i}, v_{j}\right\} \mid v_{i}, v_{j} \in V(\mathscr{G})\right.$ and $\left.i+j=n+1\right\}$ be the vertex cut sets, where $1<i<n / 2$ and $n / 2<j<n$ and clearly $|\mathscr{X}|=n / 2-2$. We assume that $\delta$ contains $X_{l}$, where $l \geq 2$. Suppose, for contradictions $l=1$, clearly $t=2$. Let $w$ be a vertex of $\mathscr{D}_{2}$ such that $E\left(X_{1},\{w\}\right)=\varnothing$ and $E(\mathcal{S},\{w\}) \neq \varnothing$. Then, there exists a vertex $x$ of $\mathcal{S}$ such that we have the following:

(i) $\mathcal{S}-\{x\}$ is connected

(ii) $X_{1} \cap\{x\}=\varnothing$

(iii) $E(\{w\},\{x\})=\varnothing$

Then, by removing vertex $x$ from $\delta$ and adding vertex $w$ in $\mathcal{S}$, we get a connected safe set $\mathcal{S}^{*}$ and for $\mathcal{S}^{*}$, we have $\left|\mathscr{D}_{1}\right|<\max \left\{\mid \mathscr{D} \| \mathscr{D} \in C\left(\mathscr{G} \backslash \mathcal{S}^{*}\right)\right\}$, which is a contradiction. Hence, $l \geq 2$. It is sufficient to show for $X_{1}$ and $X_{2}$ that $|\mathcal{S}|=c s(\mathscr{G})$. Note that if $E\left(X_{1}, X_{2}\right) \neq \varnothing$, then $t=2$. Hence, we assume that $E\left(X_{1}, X_{2}\right)=\varnothing$. Suppose that $v_{i_{1}}, v_{i_{2}} \in \mathcal{S}$ such that $E\left(\left\{v_{i_{1}}\right\}, X_{1}\right) \neq \varnothing$ and $E\left(\left\{v_{i_{2}}\right\}, X_{2}\right) \neq \varnothing$ since $\mathcal{S}$ is connected. Therefore, half vertices between $X_{1}$ and $X_{2}$ belong to $\mathcal{S}$ and the other half belongs to a component of $\mathscr{G} \backslash \mathcal{S}\left(\right.$ say $\left.\mathscr{D}_{1}\right)$. This implies $\left|\mathscr{D}_{1}\right| \leq|\mathcal{S}|-4=\lfloor n / 4\rfloor-3$. Note that $\left|\mathscr{D}_{2}\right| \leq\left|\mathscr{D}_{3}\right|$. Thus, $\left|\mathscr{D}_{3}\right| \geq\left(n-|\mathcal{S}|-\left|\mathscr{D}_{1}\right|\right) / 2=(n-2$ $\lfloor n / 4\rfloor+2) / 2 \geq|\mathcal{S}|$, a contradiction. Hence, $|\mathcal{S}|=\lfloor n / 4\rfloor+2$.

The similar arguments might work for other cases.

\section{Safe Set of the Wheel Graph}

For convenience, we consider the wheel graph $\mathscr{G}$ of order $n \geq 4$ with vertex set $V(\mathscr{G})=\left\{v_{1}, v_{2}, \ldots, v_{n}\right\}$ labeled as shown in Figure 2 [14]. 


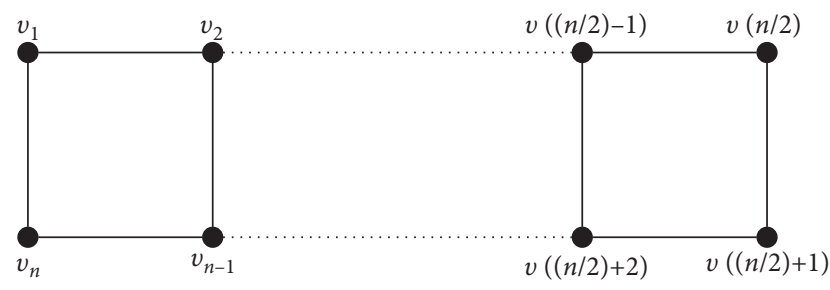

Figure 1: The ladder graph $\mathscr{G}$.

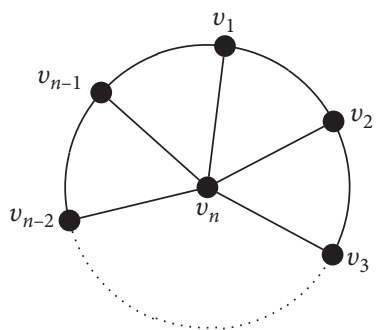

Figure 2: The wheel graph $\mathscr{G}$.

Theorem 3. Let $\mathscr{G}$ be a wheel graph. Then,

$$
\mathcal{S}=\left\{v_{1}, v_{1+k}, v_{1+2 k}, \ldots, v_{1+(k-3) k}, v_{1+(k-4) k}, v_{j}, v_{n}\right\},
$$

where

$$
v_{j}= \begin{cases}v_{n-1}, & \text { if } 2+(k-3) k \geq n, \\ v_{1+(k-3) k}, & \text { otherwise, }\end{cases}
$$

where $k=\lceil\sqrt{n}\rceil+1$ and $1+(k-4) k<j<n$.

Proof 3. Let $\mathcal{S}=\left\{v_{1}, v_{1+k}, v_{1+2 k}, \ldots, v_{1+(k-3) k}, v_{1+(k-4) k}\right.$, $v_{j}, v_{n}$ \} be a subset of $V(\mathscr{G})$. Since $\operatorname{deg}\left(v_{n}\right)=n-1$, this implies $\mathscr{G}[\mathcal{S}]$ is connected. Therefore, $C(\mathscr{G} \backslash \mathcal{S})=\left\{\mathscr{D}_{1}\right.$, $\left.\mathscr{D}_{2}, \ldots, \mathscr{D}_{l}\right\}$, where $\mathscr{D}_{1}=\left\{v_{2}, v_{3}, \ldots, v_{k}\right\}, \mathscr{D}_{2}=\left\{v_{2+k}, v_{3+k}\right.$, $\left.\ldots, v_{2 k}\right\}, \ldots, \mathscr{D}_{l-2}=\left\{v_{2+(k-5) k}, v_{3+(k-5) k}, \ldots, v_{(k-4) k}\right\}, \mathscr{D}_{l-1}=$ $\left\{v_{2+(k-4) k}, v_{3+(k-4) k}, \ldots, v_{j-1}\right\}$, and $\mathscr{D}_{l}=\left\{v_{j+1}, v_{j+2}, \ldots, v_{n-1}\right\}$. Now, $\left|\mathscr{D}_{i}\right|=k-1=\lceil\sqrt{n}\rceil=|\mathcal{S}|$ for all $1 \leq i \leq l-2$. Hence, we have two cases:

Case 1 : let $2+(k-3) k \geq n$; then, $v_{j}=v_{n-1}$; this implies $\mathscr{D}_{l}=\varnothing$. Hence, $\mathscr{D}_{l-1}=\left\{v_{2+(k-4) k}, v_{3+(k-4) k}, \ldots, v_{n-2}\right\}$ and $\quad\left|\mathscr{D}_{l-1}\right|=n-(k-4) k-3=n-([\sqrt{n}\rceil)^{2}+$ $2(\lceil\sqrt{n}\rceil)-3$.

Case 2: let $2+(k-3) k<n$; then, $v_{j}=v_{1+(k-3) k}$; this implies $\mathscr{D}_{l-1}=,\left\{v_{2+(k-4) k}, v_{3+(k-4) k}, \ldots, v_{(k-3) k}\right\}$ and $\mathscr{D}_{l}=\left\{v_{2+(k-3) k}, v_{3+(k-3) k}, \ldots, v_{n-1}\right\}$. Therefore, $\left|\mathscr{D}_{l-1}\right|=$ $k-1$ and $\left|\mathscr{D}_{l}\right|=n-(k-3) k-2=n-(\lceil\sqrt{n}\rceil)^{2}+$ $(\lceil\sqrt{n}\rceil)$.

It is clear from both the cases that $\left|\mathscr{D}_{i}\right| \leq|\mathcal{S}|$ for all $i=1,2, \ldots, l$. Thus, $\mathcal{S}$ is a connected safe set.

Theorem 4. For a wheel graph $\mathscr{G}$, the following holds:

$$
\operatorname{cs}(\mathscr{G})=\lceil\sqrt{n}\rceil,
$$

where $n$ is the order of $\mathscr{G}$.

Proof 4. Let $\mathcal{S}$ be the connected safe set of size cs $(\mathscr{G})$; then, $C(\mathscr{G} \backslash \mathcal{S})=\left\{\mathscr{D}_{1}, \mathscr{D}_{2}, \ldots, \mathscr{D}_{t}\right\}$, ordered so that $\left|\mathscr{D}_{1}\right| \leq$ $\left|\mathscr{D}_{2}\right| \leq \ldots \leq\left|\mathscr{D}_{t}\right|$.

Note that $v_{n} \in V(\mathscr{G})$ is a vertex such that $\operatorname{deg}(v)=n-1$. If $t=1$, then we have the following two cases:

Case 1: if $v_{n} \notin \mathcal{S}$, then $\mathcal{S} \subset\left(V(\mathscr{G})-\left\{v_{n}\right\}\right.$. Since $\mathcal{S}$ is connected, $V(\mathscr{G})-\left\{v_{n}\right\}$ is the cycle. Then, clearly $\mathscr{G}[\mathcal{S}]$ is a path. Let $u \in \mathcal{S} \operatorname{such}$ that $\operatorname{deg}(u)=2$ in $\mathscr{G}[\mathcal{S}]$. Then, by removing vertex $u$ from $\mathcal{S}$ and adding vertex $v_{n}$ in $\mathcal{S}$, we get a connected safe set $\mathcal{S}^{*}$ for $\mathcal{S}^{*}\left|\mathscr{D}_{1}\right|<$ $\max \left\{\mid \mathscr{D} \| \mathscr{D} \in C\left(\mathscr{G} \backslash \mathcal{S}^{*}\right)\right\}$.

Case 2: if $v_{n} \in \mathcal{S}$, then a vertex $u$ exists in $\mathcal{S}$ for which $E\left(\{u\}, \mathscr{D}_{1}\right)=\varnothing$. Let $y$ be a vertex of $D_{1}$ such that $E(\{y\}, \mathcal{S}) \neq \varnothing$. Then, by removing vertex $u$ from $\mathcal{S}$ and adding vertex $y$ in $\mathcal{S}$, we get a connected safe set $\mathcal{S}^{*}$ and for $\mathcal{S}^{*}$ and we have $\left|\mathscr{D}_{1}\right|<\max \left\{\mid \mathscr{D} \| \mathscr{D} \in C\left(\mathscr{G} \backslash \mathcal{S}^{*}\right)\right\}$.

Both cases show contradiction. Hence, $t \geq 2$.

If we choose $\mathcal{S}$ in such a way that for all $x^{\prime}, y^{\prime} \in S$, $x^{\prime} y^{\prime} \notin E\left(\mathscr{G}[\mathcal{S}]-\left\{v_{n}\right\}\right)$, then $t=|\mathcal{S}|-2$. Note that this is the most possible case of choosing the connected safe set $\delta$ for which $\mathscr{G} \backslash \mathcal{S}$ has maximum components. As a result, we have $2 \leq t \leq|\mathcal{S}|-2$.

We want to prove that $c s(\mathscr{G})=\lceil\sqrt{n}\rceil$. On the contrary, assume that $\operatorname{cs}(\mathscr{G})=\lceil\sqrt{n}\rceil-1$. Note that $t=\lceil\sqrt{n}\rceil-2$. We assume that $\left|\mathscr{D}_{i}\right|=|\mathcal{S}|$ for all $1 \leq i \leq t$, so $|V(\mathscr{G})|=$ $\sum_{i=1}^{t}\left|\mathscr{D}_{i}\right|+|\mathcal{S}|=(\lceil\sqrt{n}\rceil-2)(\lceil\sqrt{n}\rceil-$

$1)+\lceil\sqrt{n}\rceil-1=(\lceil\sqrt{n}\rceil-1)^{2}<n$, a contradiction, and consequently, the cardinality of at least one component of $\mathscr{G} \backslash \mathcal{S}$ must be greater than $|\mathcal{S}|$, which is impossible. Hence, $\operatorname{cs}(\mathscr{G})=\lceil\sqrt{n}\rceil$.

\section{Safe Set of the Sunlet Graph}

For convenience, we considered the sunlet graph $\mathscr{G}$ of order $n \geq 6$ with vertex set $V(\mathscr{G})=\left\{v_{1}, v_{2}, \ldots, v_{n}\right\}$ labeled as shown in Figure 3 [15].

Theorem 5. Let $\mathscr{G}$ be a sunlet graph of order $n \geq 6$ and $S$ be the connected safe set; then,

$$
\mathcal{S}=\left\{v_{2}, v_{4}, \ldots, v_{2\lfloor n / 3\rfloor}\right\}
$$

Proof 5. Suppose that $\mathcal{S}=\left\{v_{2}, v_{4}, \ldots, v_{2\lfloor n / 3\rfloor}\right\}$ is a subset of $V(\mathscr{G})$ such that $\mathscr{G}[\mathcal{S}]$ is its induced subgraph. For $v_{i}, v_{i+2} \in \mathcal{S}, N\left[v_{i}\right] \cap N\left[v_{i+2}\right]=\left\{v_{i}, v_{i+2}\right\}$, where $i=2,4, \ldots$, $2\lfloor n / 3\rfloor-2$. Hence $\mathscr{G}[\mathcal{S}]$ is connected.

We want to show that $\mathcal{S}$ is a connected safe set. Thus, $C(\mathscr{G} \backslash \mathcal{S})=\left\{\mathscr{D}_{1}, \mathscr{D}_{2}, \ldots, \mathscr{D}_{t}\right\}$, where $\mathscr{D}_{1}=\left\{v_{1}\right\}, \mathscr{D}_{2}=\left\{v_{3}\right\}$, $\ldots, \mathscr{D}_{t-1}=\left\{v_{2\lfloor n / 3\rfloor-1}\right\}$ and $\mathscr{D}_{t}=\left\{v_{2\lfloor n / 3\rfloor+1}, v_{2\lfloor n / 3\rfloor+2}, \ldots, v_{n}\right\}$. Note that $\left|\mathscr{D}_{j}\right|<|\mathcal{S}|$ for all $1 \leq j \leq t-1$. Now, $\left|\mathscr{D}_{t}\right|=n-2\lfloor n / 3\rfloor \leq|\mathcal{S}|$. Hence, $\mathcal{S}$ is a connected safe set.

Theorem 6. For a sunlet graph $\mathscr{G}$ of order $n \geq 6$, the following holds: 




FIgURE 3: The sunlet graph $\mathscr{G}$.

$$
\operatorname{cs}(\mathscr{G})=\left\lfloor\frac{n}{3}\right\rfloor
$$

Proof 6. Assume that $\mathcal{S}$ is a connected safe set of cardinality $\operatorname{cs}(\mathscr{G})$; then, $C(\mathscr{G} \backslash \mathcal{S})=\left\{\mathscr{D}_{1}, \mathscr{D}_{2}, \ldots, \mathscr{D}_{t}\right\}$, ordered such that $\left|\mathscr{D}_{1}\right| \leq\left|\mathscr{D}_{2}\right| \leq \ldots \leq\left|\mathscr{D}_{k}\right|$.

If $t=1$, then $\mathcal{S}=X \cup Y$, where $X=\{v \mid v \in \mathcal{S}$ and deg $(v)=3\}$ and $Y=\{u \mid u \in \mathcal{S}$ and $\operatorname{deg}(v)=1\}$. Note that $\mathcal{S}-$ $\{v\}$ is disconnected for all $v \in X$. Let $u \in Y$ and $w \in \mathscr{D}_{1}$ such that $E(\{w\}, \mathcal{S}) \neq \varnothing$; then, $\mathcal{S}^{*}=(\mathcal{S}-\{u\}) \cup\{w\}$ is a connected safe set and for $\mathcal{S}^{*},\left|\mathscr{D}_{1}\right|<\max \left\{|\mathscr{D}| \mid \mathscr{D} \in C\left(\mathscr{G} \backslash \mathcal{S}^{*}\right)\right\}$, a contradiction. Thus, $t \geq 2$.

Let $Z \subset V(\mathscr{G})$ such that $Z \cap \mathcal{S}=\varnothing, E(\mathcal{S}, Z) \neq \varnothing$, and $\mathscr{G}[Z]$ is a path. Note that $\mathcal{S}^{\prime}=(\mathcal{S}-Y) \cup Z$ is a connected safe set; then, obviously $\mathscr{G}[\mathcal{S}]$ is the path, and for that choice of the connected safe set, we have $\left|C\left(\mathscr{G} \backslash \mathcal{S}^{\prime}\right)\right|=$ Max $\{|C(\mathscr{G} \backslash \mathcal{S})| \mid S$ is a connected safe set $\}$. As a result, $2 \leq$ $t \leq|\mathcal{S}|+1$.

Suppose on the contrary that $c s(\mathscr{G})=\lfloor n / 3\rfloor-1$. Since $2 \leq t \leq|\mathcal{S}|+1, \quad d=\sum_{i=1}^{k-1}\left|\mathscr{D}_{i}\right| \leq|\mathcal{S}|$ and $n-2\lfloor n / 3\rfloor+2 \geq$ $|V(\mathscr{G})-\mathcal{S}-d|=\left|\mathscr{D}_{k}\right|>|S|$, a contradiction. Hence, $\operatorname{cs}(\mathscr{G})=\lfloor n / 3\rfloor$.

\section{Conclusion}

In this article, the connected safe set and connected safe number of ladder, wheel, and sunlet graphs are studied. The computation of connected safe number is an NP-complete problem and is known for few classes of graphs. A nontrivial graph of order $n$ whose degree set consists of $n-1$ elements is called an antiregular graph. Hence, in the future, it is interesting to study the safe set number for some other standard classes of graphs such as regular graphs, antiregular graphs, and well-known computer networks.

\section{Data Availability}

No data were used to support this study.

\section{Conflicts of Interest}

The authors declare that they have no conflicts of interest.

\section{References}

[1] B. Korte and J. Vygen, Combinatorial Optimization, SpringerVerlag, Berlin, Heidelberg, 5th edition, 2011.

[2] S. Fujita, G. MacGillivray, and T. Sakuma, "Safe set problem on graphs," Discrete Applied Mathematics, vol. 215, pp. 106$111,2016$.

[3] G. Chartrand, L. Lesniak, and P. Zhang, Graphs and Digraphs, Chapman \& Hall, London, UK, 5th edition, 2011.

[4] R. Águeda, N. Cohen, S. Fujita et al., "Safe sets in graphs: graph classes and structural parameters," Journal of Combinatorial Optimization, vol. 36, no. 4, pp. 1221-1242, 2018.

[5] B. Kang, S. Kim, and B. Park, "On safe sets of the cartesian product of two complete graphs," Ars Combinatoria, vol. 141, pp. 243-257, 2018.

[6] R. B. Bapat, S. Fujita, S. Legay et al., "Safe sets, network majority on weighted trees," Networks, vol. 71, no. 1, pp. 81-92, 2018.

[7] S. Ehard and D. Rautenbach, "Approximating connected safe sets in weighted trees," Discrete Applied Mathematics, vol. 281, pp. 216-223, 2020.

[8] S. Fujita, T. Jensen, B. Park, and T. Sakuma, "On the weighted safe set problem on paths and cycles," Journal of Combinatorial Optimization, vol. 37, no. 2, pp. 685-701, 2019.

[9] S. Fujita, B. Park, and T. Sakuma, "Stable structure on safe set problems in vertex-weighted graphs," European Journal of Combinatorics, vol. 91, Article ID 103211, 2021.

[10] R. Belmonte, T. Hanaka, I. Katsikarelis, M. Lampis, H. Ono, and Y. Otachi, "Parameterized complexity of safe set," in Proceedings of the International Conference on Algorithms and Complexity, Springer, Larnaca, Cyprus, May 2019.

[11] A. F. U. Macambira, L. Simonetti, H. Barbalho, P. H. Gonzalez, and N. Maculan, "A new formulation for the safe set problem on graphs," Computers and Operations Research, vol. 111, pp. 346-356, 2019.

[12] S. Fujita and M. Furuya, "Safe number and integrity of graphs," Discrete Applied Mathematics, vol. 247, pp. 398-406, 2018.

[13] S. N. Daoud and A. Elsonbaty, "Complexity of some graphs generated by ladder graph," International Journal of Applied Mathematics and Statistics, vol. 36, no. 6, pp. 87-94, 2013.

[14] X. Yu, L. Zhou, and X. Li, "A novel hybrid localization scheme for deep mine based on wheel graph and chicken swarm optimization," Computer Networks, vol. 154, pp. 73-78, 2019.

[15] T. N. Saibavani and N. Parvathi, "Power domination number of sunlet graph and other graphs," Journal of Discrete Mathematical Sciences and Cryptography, vol. 22, no. 6, pp. 1121-1127, 2019. 